\title{
A Hybrid Grey Based KOHONEN Model and Biogeography-Based Optimization for Project Portfolio Selection
}

\author{
Farshad Faezy Razi, ${ }^{1}$ Abbas Toloie Eshlaghy, ${ }^{2}$ Jamshid Nazemi, ${ }^{2}$ \\ Mahmood Alborzi, ${ }^{2}$ and Alireza Pourebrahimi ${ }^{3}$ \\ ${ }^{1}$ Department of Industrial Management, Semnan Branch, Islamic Azad University, Semnan, Iran \\ ${ }^{2}$ Department of Industrial Management, Science and Research Branch, Islamic Azad University, Tehran, Iran \\ ${ }^{3}$ Department of Industrial Management, Karaj Branch, Islamic Azad University, Karaj, Iran
}

Correspondence should be addressed to Abbas Toloie Eshlaghy; toloie@gmail.com

Received 22 April 2014; Revised 5 July 2014; Accepted 9 July 2014; Published 7 August 2014

Academic Editor: Han H. Choi

Copyright (C) 2014 Farshad Faezy Razi et al. This is an open access article distributed under the Creative Commons Attribution License, which permits unrestricted use, distribution, and reproduction in any medium, provided the original work is properly cited.

\begin{abstract}
The problem of selection and the best option are the main subject of operation research science in decision-making theory. Selection is a process that scrutinizes and investigates several quantitative and qualitative, and most often incompatible, factors. One of the most fundamental management issues in multicriteria selection literature is the multicriteria adoption of the projects portfolio. In such decision-making condition, manager is seeking for the best combination to build up a portfolio among the existing projects. In the present paper, KOHONEN algorithm was first employed to build up a portfolio of the projects. Next, each portfolio was evaluated using grey relational analysis (GRA) and then scheduled risk of the project was predicted using Mamdani fuzzy inference method. Finally, the multiobjective biogeography-based optimization algorithm was utilized for drawing risk and rank Pareto analysis. A case study is used concurrently to show the efficiency of the proposed model.
\end{abstract}

\section{Introduction}

One of the most important issues in project management is the selection of the project and scheduling the project related activities [1]. It selects a project or a portfolio of the existing projects. Projects can be related to research and development (R\&D) activities, information technology (IT), or investment. Selection process in such projects is affected by human, financial, and management resources limitations [2]. Today, a multicriteria selection based model, more than ever, considers several models with diversity of criteria [3]. To select a portfolio for the projects, several and various factors are taken into consideration. Those factors can be quantitative or qualitative. Quantitative factors can be directly measured, while qualitative factors are converted into numbers through ordinal scales. In the problems of selecting the projects, there are conflicting goals and measures, which should simultaneously satisfy decision maker [4]. Therefore, selecting the portfolio of the projects is a multicriterion decisionmaking problem [5]. The mathematical decision models are philosophically categorized into four main groups, namely, choice, rank, description, and sort [6]. Currently, two new categories, namely, design and portfolio models, have been added to the mentioned groups [7]. Outranking relations are based on paired comparisons in the solutions and so are applied in absolute selection problems. The novel idea is the combination of data mining, especially clustering, with multicriteria decision-making theory [8]. The main studies include fuzzy c-means model based gray relation analysis [9], KOHONEN neural network based gray relation analysis model [10], k-means algorithm based gray relation analysis [11]. The KOHONEN neural network is regarded as one of the unsupervised learning algorithms in the context of data mining, artificial neural network, and data clustering. It is an unsupervised learning algorithm, based on outranking relations. The main idea of the present paper is risk and 
rank portfolio analysis in each cluster. Therefore, the present study investigates the subject of selecting projects' portfolio with a hybrid model of multiobjective biogeography-based optimization algorithm and KOHONEN neural network based on grey relational analysis. In the present strategy, projects are first clustered through KOHONEN neural network; then, each cluster is separately ranked by the grey relational analysis, and finally the results from optimal risk and rank Pareto of the projects are analyzed and investigated, through biogeography-based optimization algorithm, for multicriteria selection. The results from biogeography-based optimization algorithm have been compared to the results from artificial bee colony algorithm, shuffled frog leaping algorithm, and firefly algorithm. The present paper is presented as follows: in the second section, research literature is given. The third section describes the grey relational analysis. The fourth section investigates and scrutinizes the KOHONEN artificial neural network. The fifth section explains biogeography-based algorithm. The sixth section discusses the risk and the way it is predicted. The approach of the present study in selecting the projects' portfolio is provided in the seventh section. The case study and conclusion are given in the eighth and ninth sections, respectively.

\section{Literatures}

Project portfolio selection literature is very wide in different organizations both from strategy and modeling and application. Almost, all organizations use the concept of project portfolio selection and are faced with it in management affairs. Different kinds of operation research and decisionmaking with multiple criteria have also been used in dealing with the project portfolio selection. Rahmani et al. have employed a hybrid method of the scenario-oriented approach based AHP and binary model in selecting IT project portfolio [12]. Amiri has used the hybrid model of AHP and TOPSIS to select the project portfolio. The main criteria in Amiris study include the company size, estimated cost, feasibility, time, technology, and eventually location [13]. Dey has taken environment protection into consideration through AHP in selecting the projects' portfolio. The structure of AHP of Dey has taken three technical, environmental, and socioeconomic factors into consideration [14]. Alidi (1996) has used a four-level AHP for ranking the industrial projects [14]. The effective factors in selecting industrial projects in the research population include budget, good relations, productivity, economy, local markets, avoiding conflict, standards, technology transfer, profitability, industrial development, peace, and environment protection. Aragonés-Beltrán et al. have employed ANP comparative approach and AHP for construction project of a solar plant [15]. Given the effects of social, political, legal, technical, and economic factors on each other, in the present study the results from ANP have been selected. Wang et al. have used a hybrid approach of ANP and GP model for information system project portfolio selection [16]. Dikmen et al. have analyzed benefits, opportunities, risk, and costs subnetworks using ANP with respect to the existing feedback within quantitative and qualitative criteria [17]. Khalili-Damghani and Sadi-Nezhad proposed GP model and
TOPSIS technique to evaluate project investment criteria [18]. San Cristóbal has used a hybrid approach of AHP and VIKTOR for selecting new energy related project [19]. Daneshvar Rouyendegh and Erol have used fuzzy ELECTRE method for project selection. Research criteria are triangular fuzzy numbers including NPV, quality, technology employed by the contractor, and economic situation [20]. Shakhsi-Niaei et al. have provided a comprehensive framework for the project portfolio selection under uncertainty conditions.

They have proposed a two-stage process based on Monte Carlo simulation technique for portfolio selection. The selection processes are based on PROMETHEE and integer programming model for efficient portfolio selection [21]. Chang and Lee have provided a hybrid model based on DEA and knapsack model as well as rough set theory for the project portfolio selection [22]. Liu and Ye have investigated the dynamic effects of time risk of the projects through system dynamic approach. In the present study, the subsystems with casual relations include plan, cost, resources, organizational relations, and strategic portfolio [23]. The main challenge in the study by Araúzo et al. is the design of a system by which resources procurement operations can optimize the life cycle in the portfolio through auction mechanism. In this study, the projects, market space, and resources are defined as the factors.

In the existing model, the factors are competing against each other for using resources through agent-based stimulation [24]. Biernatzki et al. have analyzed the issue of selection and management of energy projects using agent-based stimulation. The main factor whose behavior has been investigated in the present study includes the factors' behavior in the annual, four-month, monthly, and daily contracts [25]. Zhang has used grey relational analysis (GRA) model for analyzing investment and yield risk in order to evaluate and select investment projects. Two factors, namely, risk and yield, in the GRA have been investigated through management capability, operation, market, market exit conditions, and cost factors [26]. Mohaghar et al. have used a hybrid model of ANP and TOPSIS under fuzzy conditions to select research and development projects [27]. Vetschera and de Almeida used PROOMETHEE for projects selection. In this study, PROMETHEE V has been compared, using C-Optimal. The results show that the selection of projects using COptimal generated better output, compared to PROMETHEE $\mathrm{V}$. When the problems dimensions are smaller, the ranks of PROMETHEE V provide better results [28]. A summary of the conducted studies in the context of project portfolio selection is presented in Table 1.

\section{Grey Relational Analyses}

The grey theory was introduced by Ju-Long [29]. Grey relational analysis (GRA) model for analyzing uncertainty systems, in which part of information is unknown, was proposed. This model has had the widest application in the area of economic decisions, marketing research, modeling system, social sciences, and management [30]. The concept of grey system differs from statistics and information theory, because the former is not based on sample size and sampling methods. 
TABLE 1: A summary of the previous studies on project portfolio selection.

\begin{tabular}{|c|c|c|c|c|}
\hline Number & Decision model/method & Decision problem & Decision conditions & Researcher(s) \\
\hline 1 & AHP-BLP & IT deployment project in Tebyan & Nondeterministic & Rahmani et al., 2012 [12] \\
\hline 2 & AHP-TOPSIS & Locating project of National Oil Company & Deterministic & Amiri, 2010 [13] \\
\hline 3 & AHP & Indian oil pipelines & Deterministic & Dey, $2006[14]$ \\
\hline 4 & ANP-AHP & Construction project of solar plant & Deterministic & $\begin{array}{l}\text { Aragonés-Beltrán et al., } \\
2010 \text { [15] }\end{array}$ \\
\hline 5 & ANP-GP & Information system development project & Deterministic & Wang et al., 2009 [16] \\
\hline 6 & ANP & Turkey's highway project & Deterministic & Dikmen et al., 2007 [17] \\
\hline 7 & GP-TOPSIS & Indefinite-life projects & Deterministic & $\begin{array}{l}\text { Khalili-Damghani and } \\
\text { Sadi-Nezhad, } 2013 \text { [18] }\end{array}$ \\
\hline 8 & AHP-VIKOR & New energy projects & Deterministic & San Cristóbal, 2011 [19] \\
\hline 9 & Fuzzy ELECTRE & Project portfolio selection & Fuzzy & $\begin{array}{l}\text { Daneshvar Rouyendegh } \\
\text { and Erol, } 2012 \text { [20] }\end{array}$ \\
\hline 10 & SIMULATION PROMETHEE & Project portfolio selection & Nondeterministic & $\begin{array}{l}\text { Shakhsi-Niaei et al., } 2011 \\
\text { [21] }\end{array}$ \\
\hline 11 & Fuzzy DEA & Construction industry portfolio project & Fuzzy & Chang and Lee, 2012 [22] \\
\hline 12 & SD & Analysis of risk dynamics of project & Deterministic & Liu and Ye, 2010 [23] \\
\hline 13 & ABS & Project selection with limited resources & Deterministic & Araúzo et al., 2010 [24] \\
\hline 14 & ABS & German energy market portfolioselection & Deterministic & Biernatzki et al., 2004 [25] \\
\hline 15 & Grey Relational Analysis & Investment project selection & Deterministic & Zhang, 2012 [26] \\
\hline 16 & FAHP-FTOPSIS & Project portfolio selection & Fuzzy & Mohaghar et al., 2012 [27] \\
\hline 17 & PROMETHEE C-Optimal & Project portfolio selection & Deterministic & $\begin{array}{l}\text { Vetschera and de Almeida, } \\
2012[28]\end{array}$ \\
\hline
\end{tabular}

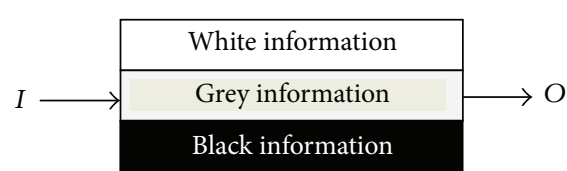

FIgURE 1: The Idea of a grey system.

In addition, it is not a fuzzy mathematical method, since it is not just seeking to deal with unknown and uncertain conditions [31]. Grey system theory has proven to be useful for dealing with problems involving poor, insufficient, and uncertain information, such as wear mode recognition [32]. The GRA possesses advantages as follows: calculations are simple and require small samples, and sample distribution is not needed as per probability theory [33]. As can be seen in Figure 1, in information theory, the dark and bright colors represent vague and clear information, respectively. In that, the black color represents the status of those systems with no absolutely certain knowledge structure, parameter, and specifications. The white color shows perfect information. The colors betweenblack and white represent vague systems [34]. The economic, social, and climate systems are of those kind.

According to Figure 1, in a grey relational system, a part of the elements is known and the rest is unknown. Analysis and grey relational analysis are important in grey theory. The differences between white, grey, and black systems are presented in Table 2 [35].
This theory in multicriteria decision-making filed is taken into consideration under uncertainty conditions, where there are complicated relations between different factors. GRA is a simple and data-oriented method in decision analysis process. Considering the issue of uncertainty, grey analysis is regarded as an appropriate method. GRA algorithms have been broadly applied for evaluating the performance of complex projects involving limited or incomplete information [36]. The grey analysis algorithm for project portfolio selection includes four fundamental steps [37].

Step 1 (definition of the grey relations). Generate the referential series of $X_{0}=\left(x_{0}(1), x_{0}(2), \ldots, x_{0}(j), \ldots, x_{0}(n)\right)$ whit $j$ enteritis, and $x_{i}$ is the compared series of $\left(x_{i}(1), x_{i}(2), \ldots, x_{i}(j), \ldots, x_{i}(n)\right)$, where $i=1,2,3, \ldots, m$ [38]. The compared series $x_{i}$ can be represented in a matrix form

$$
x_{i}=\left[\begin{array}{ccc}
x_{1}(1) & \cdots & x_{1}(n) \\
\vdots & \ddots & \vdots \\
x_{m}(1) & \cdots & x_{m}(n)
\end{array}\right] .
$$

Data can be treated by one of the three types, that is, largeris-better, smaller-is-better, and nominal-is-best. Consider the following:

$$
X_{i}(j)^{*}=\frac{x_{i}(j)-\min x_{i}(j)}{\max x_{i}(j)-\min x_{i}(j)}
$$


TABLE 2: The concept of black, grey, and white information.

\begin{tabular}{lccc}
\hline System/specifications & White & Grey & Black \\
\hline Information & Known & Incomplete & Unknown \\
Clarity & Clear & Grey & Tunnel \\
Process & Not new & The old is substituted & New \\
Trait & Regular & Complicated & Chaos \\
Methodology & Positive & Transitive & Negative \\
Trait & Accurate & Bearable & Extremist \\
conclusion & Single criterion & Multicriteria & Inconclusive \\
\hline
\end{tabular}

For the $X_{i j}$ indicator with "the more the better" criterion, the following equation is used:

$$
X_{i}(j)^{*} \frac{\max x_{i}(j)-x_{i}(j)}{\max x_{i}(j)-\min x_{i}(j)} .
$$

Finally, for the indicator "the more the index is closer to the nominal standard," the following equation is used:

$$
X_{i j}=\frac{\left|x_{i}(j)-x_{0 b}(j)\right|}{\max x_{i}(j)-x_{0 b}(j)} .
$$

Step 2 (definition of the reference sequence). The normalized referential series of $X_{0}$ becomes $x_{0}^{*}=\left(x_{0}^{*}(1), x_{0}^{*}(2), \ldots\right.$, $\left.x_{0}^{*}(n)\right)$. After the original data set is normalized by one of three types of data transformation, the matrix shown in (1) can be revised as [39]

$$
x_{i}^{*}=\left[\begin{array}{ccc}
x_{1}^{*}(1) & \cdots & x_{1}^{*}(n) \\
\vdots & \ddots & \vdots \\
x_{m}^{*}(1) & \cdots & x_{m}^{*}(1)
\end{array}\right] .
$$

Step 3 (calculation of the confidence interval for the grey relations). The confidence interval for grey relations is calculated based on the following equation:

$$
\Gamma_{0 i}(j)=\frac{\Delta_{\min +\xi \Delta_{\max }}}{\Delta_{0 i}(j)+\xi \Delta_{\max }} .
$$

$\Delta_{0 i}(j)$ is calculable by the following equation:

$$
\begin{aligned}
\Delta_{0 i}(j) & =\left|x_{0}^{*}(j)-x_{i}^{*}(j)\right| \\
& =\left[\begin{array}{ccc}
\Delta_{01}(1) & \cdots & \Delta_{01}(n) \\
\vdots & \ddots & \vdots \\
\Delta_{0 m}(1) & \cdots & \Delta_{0 m}(1)
\end{array}\right] .
\end{aligned}
$$

$\Delta_{\min }$ and $\Delta_{\max }$ are calculated based on the following equations:

$$
\begin{aligned}
& \Delta_{\min }=\min \left\{\Delta_{0 i}, i=1, \ldots, m, j=1, \ldots, n\right\}, \\
& \Delta_{\max }=\max \left\{\Delta_{0 i}, i=1, \ldots, m, j=1, \ldots, n\right\} .
\end{aligned}
$$

Step 4 (calculation of the grey relations score). To calculate the grey relations score or rank the portfolio, the following equation is used:

$$
\Gamma_{0 i}=\sum_{j=1}^{n}\left[w_{i}(j) \times \Gamma_{0 i}(j)\right]
$$

\section{KOHONEN Neural Network}

The visualization and interpretation of the KOHONEN network allows users to assume that all the samples indexed in the same neuron or in its surroundings are considered similar according to the characteristics being evaluated. This display scheme allows the easy detection of similarities in samples, such as clusters containing similar samples and outlier sample detection [40]. The structure of a KOHONEN clustering network consists of two layers, an input fan-out layer and an output (competitive) layer [41]. The SOM algorithm does not require the storage of a large number of samples, and thus it has much lower space complexity than multidimensional scaling [42]. The objective of the KOHONEN neural network is to create self-organizing classifier patterns [43]. Commonly, a KOHONEN network includes a 2D array of neurons, in which all of the inputs enter into all of the neurons. Each neuron has its own weight set, which can be regarded as the sample pattern [44]. When the network is fed with an input pattern, the neuron that has the sample pattern and the greatest similarity to the input pattern generates the highest result. One of the differences between this network and other self-organizing networks is that the sample patterns are saved in a way that the similar samples are found in physically interdependent neurons. The samples with much dissimilarity stand apart. A sample of ideal 2D arrays in KOHONEN neural network based clustered data is presented in Figure 2.

The stages of the KOHONEN neural network algorithm with assumed number of $m$ inputs and $n$ outputs are as follows:

(i) first, the initial weights of the network are randomly selected;

(ii) training samples are introduced to the network;

(iii) based on (10), every neuron of the output layer is calculated:

$$
d_{\min }=\min \left\{d_{j}=\sum_{i=1}^{n}\left(x_{i}-w_{i j}\right)^{2}, j=1, \ldots, m\right\}
$$

(iv) the winning output neurons are identified and their weights are determined using a neighborhood function, based on (11):

$$
w_{i j}(t+1)=\left(w_{i j}(t)\right)+n(t) N(t)\left(x_{i}-w_{i j}(t)\right) .
$$




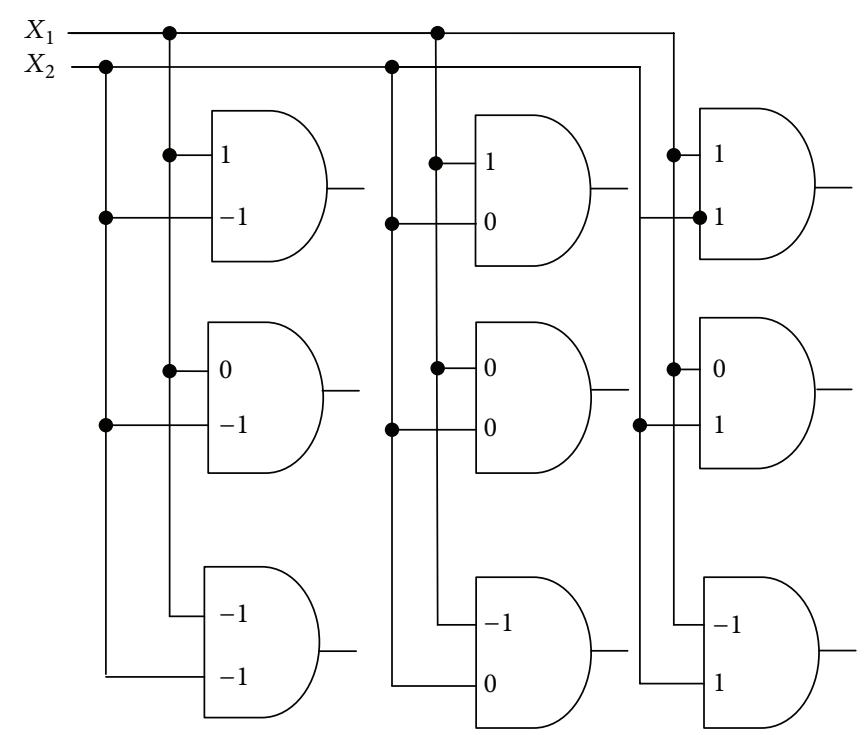

FIgURE 2: Clustering data in the KOHONEN neural network algorithm.

In (11), $n(t)$ and $N(t)$ are training and neighborhood functions, respectively.

(v) $t$-value increases.

The algorithm is repeated from the second stage. The repetition number can be regarded constant or it can continue until the neural network is trained, meaning the weight values undergo insignificant changes [45].

\section{Project Risk and a Mechanism to Evaluate It}

Portfolio risk is an uncertain event or condition that if occurred, it will positively or negatively affect one or more objectives of the project goals [46]. Every risk can be due to one or more reasons, and its impacts may affect one or more success criteria in the portfolio. The major known risks in the research population of the present study include political, environmental, technical, economic, and commercial risks. The present study has employed fuzzy inference system for predicting scheduled risk of the project. The fuzzy inference includes finding numerical answer of the numerical inputs based on a fuzzy rule. Fuzzy inference has the following stages [47].

Step 1. Measure the membership rate of the antecedents for each fuzzy set.

Step 2. Obtain the membership function of consequent fuzzy set corresponding to antecedents and obtain the cut fuzzy set.

Step 3. Aggregate the cut consequences.

Step 4. Defuzzificate the final set for obtaining the numerical answer.

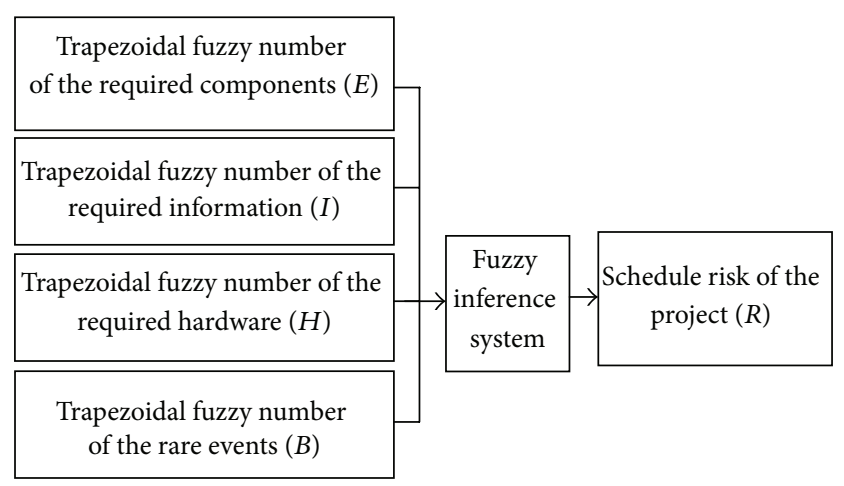

FIGURE 3: A framework for predicting the project's scheduled risk (delay risk) based on Mamdani fuzzy system.

It is worth mentioning that the above system can be in accordance to either Sugeno or Mamdani fuzzy system [48]. The major constituent factors of the project scheduled risk (delay risk) include the components required for completing a deliverable project, the information required by the project, delivery of the required hardware, and rare events or black swan phenomenon.

In the present study, Mamdani inference fuzzy system with four inputs and one output, where the delay risk is probable (with trapezoidal fuzzy number), has been used. Figure 3 shows the structure of this model.

\section{Biogeography-Based Optimization Algorithm}

The $\mathrm{BBO}$ algorithm is a population based algorithm developed for the global optimization [49]. This algorithm was introduced by Professor Dan Simon in 2008 for solving optimization problems [50]. In $\mathrm{BBO}$, each solution is modeled as a habitat, and each habitat feature or solution component is called a suitability index variable [51]. In this algorithm, unlike human, animals and plants are essentially emphasizing the exclusive use of resources. In addition, due to the lack of rules within animals, the stronger ones win. Therefore, the ecosystem causes any organism to feed on other species. In fact, tendency to monopoly in movable animals makes them emigrate to and settle in quiet places. Building on this concept, where more animal species are living is better than other places (in comparison), and so has attracted more species. In other words, HSI is higher in that area. In other words, more population would be where the habitat suitability index (HIS) is higher. In the biogeography-based optimization algorithm problems, HIS is defined as the objective function. Therefore, there is a greater propensity to emigrate to where HIS is high. The areas with low HIS have a high immigration rate. In the optimization problems, HIS is equivalent to the objective function. If the objective function was of the maximizing kind, the higher HIS would be considered and vice versa. Therefore, as can be seen in Figure 4 , if $s$ represents the population of a habitat, the emigration rate increases by the growth of population. The discussed rate has been represented with $\mu$. The emigration 


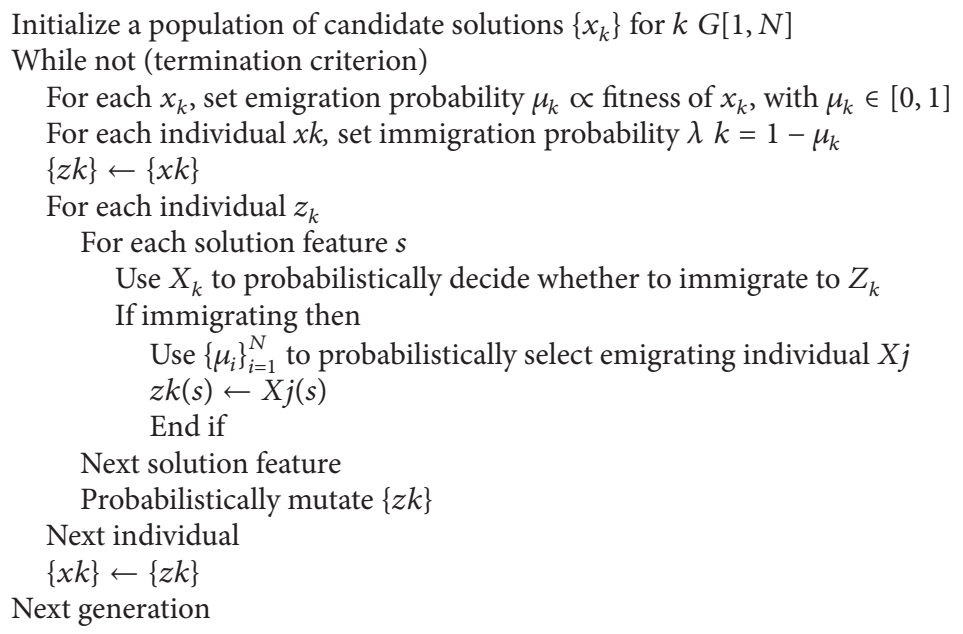

Algorithm 1

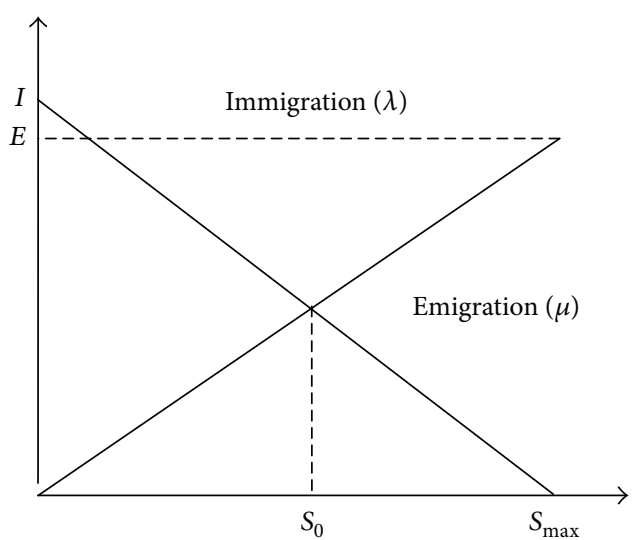

FIGURE 4: Species model of a single habitat.

rate is less in $S_{\max }$ which contains the largest number of possible states. Therefore, $S_{\max }$ has the lowest emigration rate [50]. This rule is represented by the immigration curve $(\lambda)$.

Therefore, biogeography-based optimization algorithm is shown in Algorithm 1.

\section{A Framework for the Project Portfolio Selection}

In this part of the paper, a comprehensive framework is given for project portfolio selection, using a hybrid model of $\mathrm{BBO}$ algorithm and GRA based KOHONEN network algorithm. As can be seen in Figure 5 projects are first clustered by using KOHONEN network algorithm and, based on it, they are placed in different portfolios. To select a project in a portfolio and place it in the final portfolio, the grey relational analysis is used. In other words, each cluster is independently ranked with the GRA, and then the rank and risk optimal Pareto combination are obtained through $\mathrm{BBO}$ algorithm.

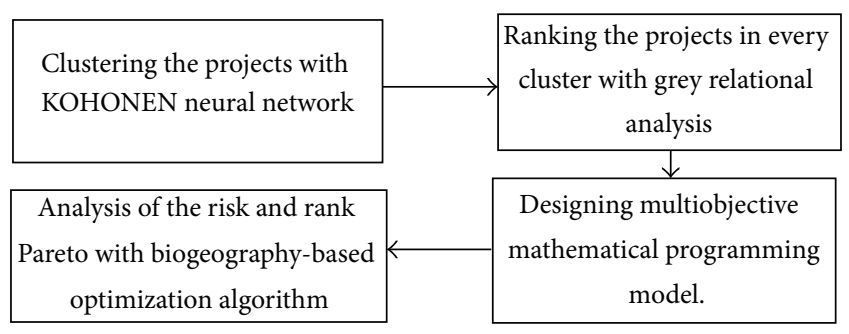

FIGURE 5: A framework for project portfolio selection using the hybrid model of biogeography-based optimization algorithm and the grey relational analysis based KOHONEN neural.

The mentioned framework has following steps.

Step 1. The initial weights of the network are selected randomly.

Step 2. Training samples are introduced to the network.

Step 3. Equation (10) is applied to all neurons of the output layer.

Step 4. The winning output neurons are determined and their weights are modified based on (11), using a neighborhood function.

Step 5. $t$-value increases in (11).

Step 6. The algorithm is repeated from the second step. The repetition number can be considered constant or can be continued until the neural network is trained, meaning the weights have undergone insignificant changes.

In this way, the projects are clustered and placed in different portfolios.

Step 7. Define the grey relations based on (3) and normalization with (4), (5), and (6). 
TABLE 3: The variables and parameters of the mathematical model of project portfolio selection.

\begin{tabular}{|c|c|}
\hline Variables of the model & Description \\
\hline One-zero variable $X_{j}$ & 1 is assigned to the $i$ th project and 0 otherwise \\
\hline$N$ & All of the proposed projects \\
\hline$r_{i}$ & Risk of every project \\
\hline$D$ & The required time for completion of each project \\
\hline$q_{i}$ & The rank of each project \\
\hline $\mathrm{AF}_{i}$ & The overall resources required \\
\hline$C_{i}$ & The resources required for the $i$ th project \\
\hline$d_{i}$ & Completion time of the $i$ th project \\
\hline \multicolumn{2}{|l|}{ Research objectives: } \\
\hline (1) minimizing the risk of portfolio & $\sum_{i=1}^{n} \sum_{j=1}^{m} r_{i} X_{j}$ \\
\hline (2) maximizing the rank of each portfolio & $\sum_{i=1}^{n} \sum_{j=1}^{m} q_{i} X_{j}$ \\
\hline \multicolumn{2}{|l|}{ Limitations: } \\
\hline (1) resources limitation for completion of the projects & $\sum_{i=1}^{n} \sum_{j=1}^{m} C_{i} X_{j}=m$ \\
\hline (2) time limitation for the completion of each project & $\sum_{i=1}^{n} \sum_{j=1}^{m} d_{i} X_{j}=D$ \\
\hline (3) binding limitations for implementation of the project & $X_{11}+X_{16} \leq 1$ \\
\hline (4) binding limitations for implementation of the project & $X_{4}-X_{8} \leq 0$ \\
\hline
\end{tabular}

Step 8. Define the reference sequence with (7).

Step 9. Calculate the confidence interval for the grey relations with (8).

Step 10. Calculate the gray relations score with (11).

Step 11. Design the mathematical programming model (the input data for designing this model are summarized in Table 3).

Step 12. Solve the model in Step 11 with biogeography-based optimization algorithm and risk and rank Pareto analysis.

\section{Case Study}

In this part of the present paper, a case study is performed to practically explain the project portfolio selection using the hybrid model of BBO algorithm and GRA based KOHONEN neural network. The input data are presented in Table 4. As can be seen in Table 4, the present value of the yield of each project $(\mathrm{C} 1)$, environmental risk (C2), the effect of project implementation on economic prosperity of the region (C3), and tariff (C4) is the criteria for 20 studied projects. For clustering the data of Table 4, KOHONEN neural network has been used. To implement this algorithm, Clementine 12.0 has been used. The result form clustering the 20 projects under investigation is presented in Figure 6. As can be seen, the number of clusters for this study, using KOHONEN neural network, is four. The third criterion, that is, the effect of project implementation of the economic prosperity of the region, is ordinal and the other scales are relative.
TABLE 4: The input data of the 20 projects under investigation.

\begin{tabular}{lcccc}
\hline Projects & Criteria 1 & Criteria 2 & Criteria 3 & Criteria 4 \\
\hline Project 1 & 10000 & 1 & 19 & 100 \\
Project 2 & 12000 & 5 & 13 & 110 \\
Project 3 & 13000 & 9 & 16 & 150 \\
Project 4 & 10000 & 3 & 18 & 100 \\
Project 5 & 15000 & 4 & 1 & 110 \\
Project 6 & 12000 & 9 & 5 & 140 \\
Project 7 & 13000 & 1 & 4 & 160 \\
Project 8 & 16000 & 9 & 2 & 130 \\
Project 9 & 14000 & 3 & 3 & 120 \\
Project 10 & 18000 & 7 & 15 & 148 \\
Project 11 & 13000 & 9 & 8 & 180 \\
Project 12 & 17000 & 3 & 20 & 135 \\
Project 13 & 15000 & 9 & 6 & 170 \\
Project 14 & 18000 & 5 & 14 & 110 \\
Project 15 & 15000 & 1 & 9 & 148 \\
Project 16 & 14000 & 7 & 17 & 170 \\
Project 17 & 12000 & 9 & 11 & 110 \\
Project 18 & 18000 & 3 & 10 & 140 \\
Project 19 & 10000 & 8 & 7 & 120 \\
Project 20 & 13500 & 8 & 12 & 115 \\
\hline
\end{tabular}

The results from the implementation of grey relational analysis for complete ranking of the projects for each cluster are summarized in Table 5.

In the present paper, Mamdani fuzzy inference system has been used for analysis and prediction of scheduled risk of the 
TABLE 5: The results from ranking the projects in each cluster with grey relational analysis.

(a)

\begin{tabular}{lcccccccccc}
\hline Project & 1 & 2 & 3 & 4 & 5 & 6 & 7 & 8 & 9 & 10 \\
Cluster & 1 & 1 & 2 & 1 & 3 & 2 & 3 & 4 & 3 & 3 \\
GRA & 0.2929 & 0.9945 & 0.5457 & 0.2925 & 0.4035 & 0.2860 & 0.2858 & 0.9989 & 0.3354 & 0.9955 \\
\hline
\end{tabular}

(b)

\begin{tabular}{lcccccccccc}
\hline Project & 11 & 12 & 13 & 14 & 15 & 16 & 17 & 18 & 19 & 20 \\
Cluster & 4 & 3 & 4 & 3 & 3 & 4 & 2 & 3 & 1 & 2 \\
GRA & 0.2858 & 0.6657 & 0.5434 & 0.9995 & 0.3997 & 0.3756 & 0.2860 & 0.9957 & 0.2857 & 0.9992 \\
\hline
\end{tabular}

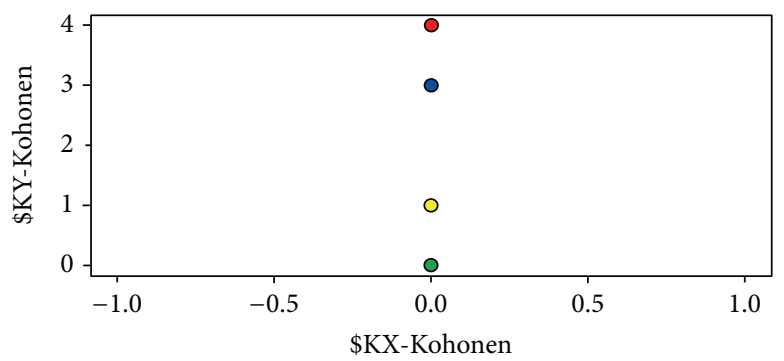

FIGURE 6: The results from implementation of the neural network.
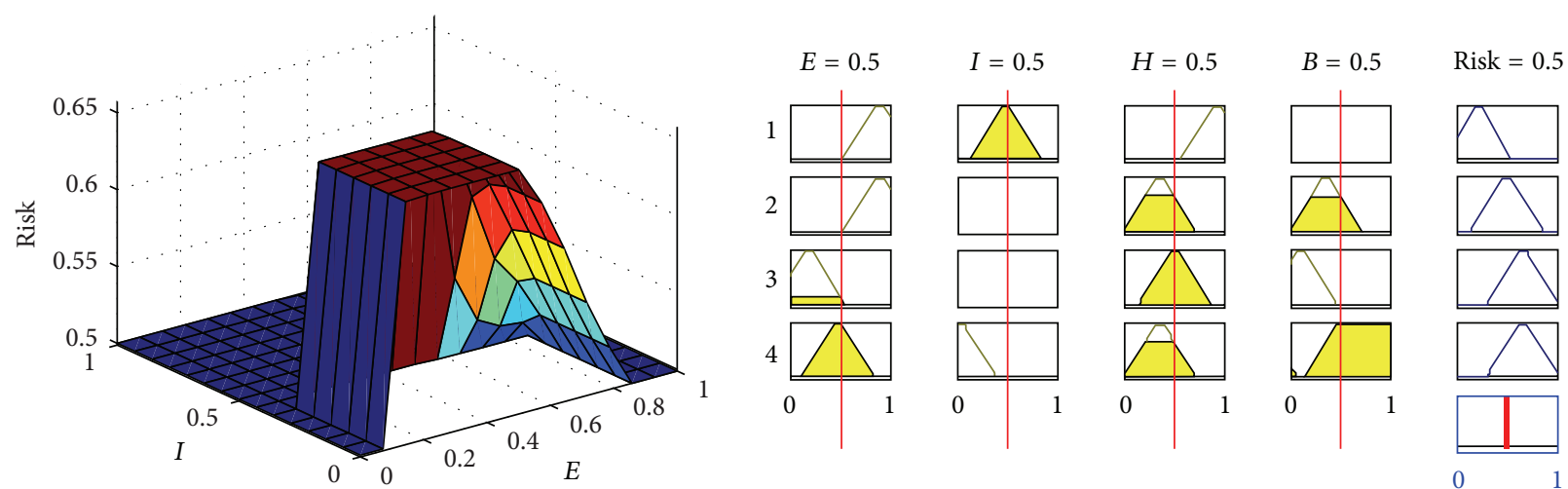

FIGURE 7: The results from prediction of scheduled risk of the first project with Mamdani-type fuzzy system.

project. The fuzzy rules for risk prediction have the following structure, with respect to the risk section in this paper.

If $E$ is good and $I$ is medium and $H$ is good then risk is low.

If $E$ is good and $B$ is medium and $H$ is low then risk is medium.

If $E$ is poor and $B$ is low and $H$ is medium then risk is high.

If $E$ is medium and $I$ is low and $H$ is low then risk is high.

For example, the result from the implementation of Mamdani-type fuzzy system for the project 1 is presented in Figure 7.
Biobjective mathematical programming model of the present study contains the following structure:

Max

$$
\begin{aligned}
Z_{1}= & 0.2929 x_{1}+0.9945 x_{2}+0.5457 x_{3} \\
& +0.2925 x_{4}+0.4035 x_{5}+0.2860 x_{6} \\
& +0.2858 x_{7}+0.9989 x_{8}+0.3353 x_{9} \\
& +0.9955 x_{10}+0.2858 x_{11}+0.6657 x_{12} \\
& +0.5434 x_{13}+0.9995 x_{14}+0.3997 x_{15} \\
& +0.3756 x_{16}+0.2860 x_{17}+0.9957 x_{18} \\
& +0.2857 x_{19}+0.9992 x_{20},
\end{aligned}
$$

Min

$$
\begin{aligned}
Z_{2}= & 0.5 x_{1}+0.42 x_{2}+0.6 x_{3}+0.55 x_{4} \\
& +0.88 x_{5}+0.34 x_{6}+0.65 x_{7}
\end{aligned}
$$



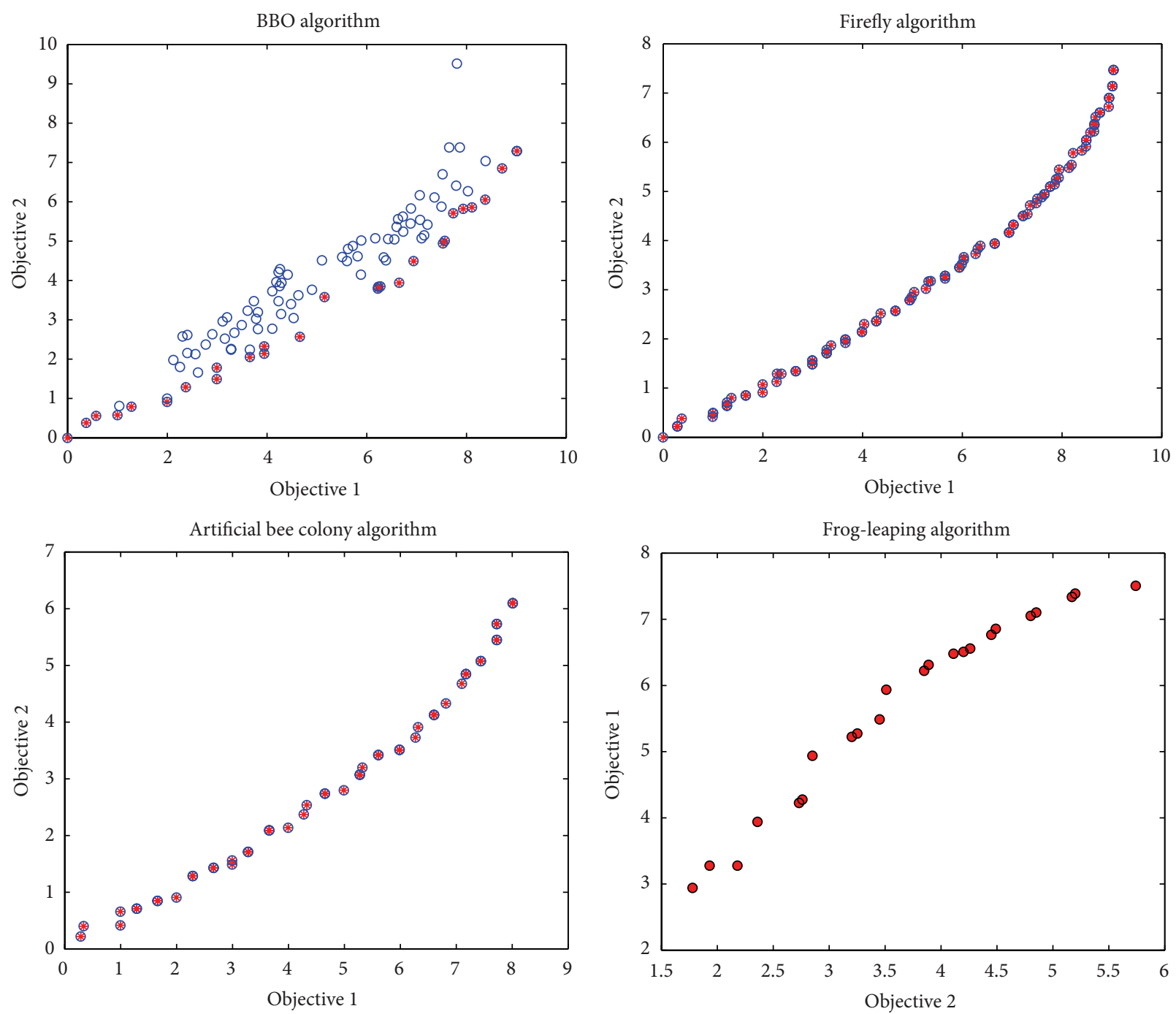

FIgURE 8: The result of the risk and rank optimal Pareto.

$$
\begin{aligned}
& +0.58 x_{8}+0.40 x_{9}+0.66 x_{10} \\
& +0.35 x_{11}+0.43 x_{12}+0.69 x_{13} \\
& +0.49 x_{14}+0.75 x_{15}+0.38 x_{16} \\
& +0.22 x_{17}+0.71 x_{18}+0.37 x_{19} \\
& +0.65 x_{20}
\end{aligned}
$$

s.t:

$$
\begin{aligned}
& 100 x_{1}+150 x_{2}+200 x_{3}+170 x_{4} \\
& +300 x_{5}+250 x_{6}+180 x_{7}+270 x_{8} \\
& +140 x_{9}+190 x_{10}+260 x_{11}+240 x_{12} \\
& +120 x_{13}+240 x_{14}+290 x_{15}+310 x_{16} \\
& +160 x_{17}+170 x_{18}+200 x_{19} \\
& +130 x_{20} \leq 2400 \\
& 90 x_{1}+150 x_{2}+300 x_{3}+280 x_{4} \\
& +500 x_{5}+460 x_{6}+360 x_{7}+420 x_{8} \\
& +190 x_{9}+280 x_{10}+470 x_{11}+350 x_{12}
\end{aligned}
$$

$$
\begin{aligned}
& +470 x_{13}+260 x_{14}+370 x_{15} \\
& +560 x_{16}+380 x_{17}+180 x_{18} \\
& +170 x_{19}+400 x_{20} \leq 3800, \\
& x_{11}+x_{16} \leq 1, \\
& x_{4}-x_{8} \leq 0, \\
& x_{j} \in\{0,1\}, \quad j=1,2,3, \ldots, 20 .
\end{aligned}
$$

The results from solving the above model with biogeography-based optimal algorithm, firefly algorithm, artificial bee colony algorithm, and frog-leaping algorithm are, respectively, presented in Figure 8.

The time required for solving by each algorithm and the value of each function is presented in Table 6 .

The adjustments related to the parameters of the algorithm implemented in the present study are summarized in Table 7. 
TABLE 6: The criteria for the result in metaheuristic algorithm used in the present study.

\begin{tabular}{lccc}
\hline Algorithm & Time & Value of first objective & Value of second objective \\
\hline Biogeography based optimization & 2621.2721 & 9.0645 & 7.1240 \\
Firefly algorithm & 631.0303 & 9.0303 & 7.9000 \\
Artificial bee colony algorithm & 7.7100 & 8.0825 & 7.7100 \\
Shuffled frog leaping algorithm & 35.1560 & 7.345 & 5.5465 \\
\hline
\end{tabular}

TABLE 7: The main adjusted parameters in the investigated algorithms.

\begin{tabular}{|c|c|c|c|c|c|c|c|}
\hline $\begin{array}{l}\text { Biogeography-based } \\
\text { optimization }\end{array}$ & Value & Firefly algorithm & Value & $\begin{array}{l}\text { Artificial bee colony } \\
\text { algorithm }\end{array}$ & Value & $\begin{array}{c}\text { Shuffled frog leaping } \\
\text { algorithm }\end{array}$ & Value \\
\hline Number of habitats & 100 & Number of fireflies & 100 & Number of bees & 100 & $\begin{array}{l}\text { Number of } \\
\text { memeplexes }\end{array}$ & 5 \\
\hline Emigration rates & 0.7 & $\begin{array}{l}\text { Light absorption } \\
\text { coefficient }\end{array}$ & 1 & Amount of food & Round (NB/2) & $\begin{array}{l}\text { Number of frogs in } \\
\text { each memeplex }\end{array}$ & 10 \\
\hline Immigration rates & 0.3 & $\begin{array}{l}\text { Attraction coefficient } \\
\text { base value }\end{array}$ & 2 & Limit & 20 & $\begin{array}{l}\text { Maximum of } \\
\text { generation }\end{array}$ & 100 \\
\hline Keep rate & 0.2 & Mutation coefficient & 0.9 & Maximum of iteration & 100 & $\begin{array}{l}\text { Iterations within each } \\
\text { memeplex }\end{array}$ & 20 \\
\hline
\end{tabular}

\section{Conclusions}

Projects portfolio multicriteria selection is a mathematical model in multicriteria decision-making theory. The main goal in this model is selecting an optimal portfolio combination from within the existing projects regarding qualitative and quantitative objectives. In multicriteria decision-making models, the issue of selection is limited to choosing a decision option. Regarding the matter of selecting a portfolio from the projects, the selection method is not in accordance with the goal of such action. Therefore, in project portfolio selection there is no multicriteria decision-making model capable of choosing the best combination from decision options (projects). The present study has been provided to solve the problem of selecting a compound from within decision options (projects) in multicriteria decision-making theory. In this paper, the idea is formation of the primary portfolio from the decision options (projects) via Kohonen neural network. After clustering with Kohonen network, decision options have been ordered in four clusters. Each of them was ranked separately through gray relation analysis. For the purpose of risk analysis and prediction of each of the 20 projects, fuzzy inferential system has been used. Results from fuzzy inferential system and gray relation analysis have been modeled in a two-objective mathematical planning model. The discussed zero and one two-objective model is obtained via biography-based optimization algorithm, firefly model, particle swarm optimization, and leap-frog method. Results show that firefly algorithm has superiority over other algorithms in terms of rate and optimality. In the present study, for selecting project portfolio, decision theory, data mining, mathematical modeling, fuzzy inferential system, and metaheuristic system were all combined together. The provided methodology in this paper is theoretically and practically applicable in all multicriteria selection problems, where the aim is formation of a portfolio.

\section{Conflict of Interests}

The authors declare that there is no conflict of interests regarding the publication of this paper.

\section{References}

[1] Y. Shou and Y. Huang, "Combinatorial auction algorithm for project portfolio selection and scheduling to maximize the net present value," Journal of Zhejiang University: Science C, vol. 11, no. 7, pp. 562-574, 2010.

[2] S. B. Graves, J. L. Ringuest, and A. L. Medaglia, Models \& Methods for Project Selection: Concepts from Management Science, Finance and Information Technology, vol. 58, Springer, 2003.

[3] I. Zelinka, V. Snasel, and A. Abraham, Handbook of Optimization: From Classical to Modern Approach, vol. 38, Springer, New York, NY, USA, 2012.

[4] F. Zandi and M. Tavana, "A multi-attribute group decision support system for information technology project selection," International Journal of Business Information Systems, vol. 6, no. 2, pp. 179-199, 2010.

[5] M. Yurdakul and Y. T. Iç, "Application of correlation test to criteria selection for multi criteria decision making (MCDM) models," International Journal of Advanced Manufacturing Technology, vol. 40, no. 3-4, pp. 403-412, 2009.

[6] B. Roy, Multicriteria Methodology for Decision Aiding, Volume 12, Springer, 1996.

[7] V. Belton and T. Stewart, Multiple Criteria Decision Analysis: An Integrated Approach, Springer, New York, NY, USA, 2002.

[8] Y. de Smet and S. Eppe, "Multicriteria relational clustering: the case of binary outranking matrices," in Evolutionary MultiCriterion Optimization, Springer, New York, NY, USA, 2009.

[9] A. T. E. Farshad Faezy Razi, J. Nazemi, M. Alborzi, and A. Poorebrahimi, "A hybrid grey based fuzzy C-means and multiple objective genetic algorithms for project portfolio selection," 
International Journal of Industrial and Systems Engineering. In press.

[10] F. F. Razi and A. T. Eshlaghy, "A hybrid grey-based KOHONEN and genetic algorithm to integrated technology selection," International Journal of Industrial and Systems Engineering. In press.

[11] F. F. R. Abbas Toloie Eshlaghy, "A hybrid Grey-based K-means and genetic algorithm for project selection," International Journal of Business Information Systems. In press.

[12] N. Rahmani, A. Talebpour, and T. Ahmadi, "Developing a Multi criteria model for stochastic IT portfolio selection by AHP method," Procedia-Social and Behavioral Sciences, vol. 62, pp. 1041-1045, 2012.

[13] M. P. Amiri, "Project selection for oil-fields development by using the AHP and fuzzy TOPSIS methods," Expert Systems with Applications, vol. 37, no. 9, pp. 6218-6224, 2010.

[14] P. K. Dey, "Integrated project evaluation and selection using multiple-attribute decision-making technique," International Journal of Production Economics, vol. 103, no. 1, pp. 90-103, 2006.

[15] P. Aragonés-Beltrán, F. Chaparro-González, J. P. PastorFerrando, and F. Rodríguez-Pozo, "An ANP-based approach for the selection of photovoltaic solar power plant investment projects," Renewable and Sustainable Energy Reviews, vol. 14, no. 1, pp. 249-264, 2010.

[16] J. Wang, Y. Xu, and Z. Li, "Research on project selection system of pre-evaluation of engineering design project bidding," International Journal of Project Management, vol. 27, no. 6, pp. 584-599, 2009.

[17] I. Dikmen, M. T. Birgonul, and B. Ozorhon, "Project appraisal and selection using the analytic network process," Canadian Journal of Civil Engineering, vol. 34, no. 7, pp. 786-792, 2007.

[18] K. Khalili-Damghani and S. Sadi-Nezhad, "A hybrid fuzzy multiple criteria group decision making approach for sustainable project selection," Applied Soft Computing Journal, vol. 13, no. 1, pp. 339-352, 2013.

[19] J. San Cristóbal, "Multi-criteria decision-making in the selection of a renewable energy project in spain: the Vikor method," Renewable Energy, vol. 36, no. 2, pp. 498-502, 2011.

[20] B. Daneshvar Rouyendegh and S. Erol, "Selecting the best project using the fuzzy ELECTRE method," Mathematical Problems in Engineering, vol. 2012, Article ID 790142, 12 pages, 2012.

[21] M. Shakhsi-Niaei, S. A. Torabi, and S. H. Iranmanesh, "A comprehensive framework for project selection problem under uncertainty and real-world constraints," Computers and Industrial Engineering, vol. 61, no. 1, pp. 226-237, 2011.

[22] P. Chang and J. Lee, "A fuzzy DEA and knapsack formulation integrated model for project selection," Computers \& Operations Research, vol. 39, no. 1, pp. 112-125, 2012.

[23] R. Liu and Z. Ye, "System dynamics application in analyzing the schedule risk influence on project portfolio," in Proceedings of the 8th International Conference on Supply Chain Management and Information Systems: Logistics Systems and Engineering (SCMIS '10), October 2010.

[24] J. A. Araúzo, J. Pajares, and A. Lopez-Paredes, "Simulating the dynamic scheduling of project portfolios," Simulation Modelling Practice and Theory, vol. 18, no. 10, pp. 1428-1441, 2010.

[25] R. Biernatzki, B. Bitzer, H. Convey, and A. J. Hartley, "Agent based simulation system for portfolio management in deregulated energy markets," in Proceeding of the 39th International
Universities Power Engineering Conference (UPEC '04), vol. 2, pp. 1145-1149, Bristol, UK, September 2004.

[26] X. Zhang, "Venture capital investment base on grey relational theory," Physics Procedia, vol. 33, pp. 1825-1832, 2012.

[27] A. Mohaghar, M. R. Fathi, A. Faghih, and M. M. Turkayesh, "An integrated approach of Fuzzy ANP and Fuzzy TOPSIS for R\&D project selection: a case study," Australian Journal of Basic and Applied Sciences, vol. 6, no. 2, pp. 66-75, 2012.

[28] R. Vetschera and A. T. de Almeida, "A PROMETHEE-based approach to portfolio selection problems," Computers and Operations Research, vol. 39, no. 5, pp. 1010-1020, 2012.

[29] D. Ju-Long, "Control problems of grey systems," Systems \& Control Letters, vol. 1, no. 5, pp. 288-294, 1982.

[30] T. Gwo-Hshiung, G. H. Tzeng, and J.-J. Huang, Multiple Attribute Decision Making: Methods and Applications, CRC Press, 2011.

[31] P. Mujumdar and S. Karmakar, "Grey fuzzy multi-objective optimization," in Fuzzy Multi-Criteria Decision Making, pp. 453-482, Springer, 2008.

[32] Y. F. Wang, S. Chen, Y. Lee, and C. Tsai, "Developing green management standards for restaurants: an application of green supply chain management," International Journal of Hospitality Management, vol. 34, no. 1, pp. 263-273, 2013.

[33] S. Goyal and S. Grover, "Applying fuzzy grey relational analysis for ranking the advanced manufacturing systems," Grey Systems: Theory and Application, vol. 2, no. 2, pp. 284-298, 2012.

[34] A. Samvedi, V. Jain, and F. T. Chan, "An integrated approach for machine tool selection using fuzzy analytical hierarchy process and grey relational analysis," International Journal of Production Research, vol. 50, no. 12, pp. 3211-3221, 2012.

[35] K. Chang, Y. Chang, and I. Tsai, "Enhancing FMEA assessment by integrating grey relational analysis and the decision making trial and evaluation laboratory approach," Engineering Failure Analysis, vol. 31, pp. 211-224, 2013.

[36] A. Saavedra, E. Arce, J. L. Miguez et al., "Potential effect of uncertainty on the GRG interpretation," Grey Systems: Theory and Application, vol. 3, no. 2, pp. 121-128, 2013.

[37] K. Palanikumar, B. Latha, V. S. Senthilkumar, and J. P. Davim, "Analysis on drilling of glass fiber-reinforced polymer (GFRP) composites using grey relational analysis," Materials and Manufacturing Processes, vol. 27, no. 3, pp. 297-305, 2012.

[38] H. Wu, "A comparative study of using grey relational analysis in multiple attribute decision making problems," Quality Engineering, vol. 15, no. 2, pp. 209-217, 2002.

[39] J. H. Wu and C. Chen, "An alternative form for grey correlative grades," The Journal of Grey System, vol. 11, no. 1, pp. 7-12, 1999.

[40] F. A. Ribeiro, F. F. Rosário, M. C. M. Bezerra et al., "Evaluation of chemical composition of waters associated with petroleum production using Kohonen neural networks," Fuel, vol. 117, pp. 381-390, 2014.

[41] C. W. D. de Almeida, R. M. C. R. de Souza, and A. L. B. Candeias, "Fuzzy Kohonen clustering networks for interval data," Neurocomputing, vol. 99, pp. 65-75, 2013.

[42] A. S. Sunay, Ù. Cunediòlu, and B. Ylmaz, "Feasibility of probabilistic neural networks, Kohonen self-organizing maps and fuzzy clustering for source localization of ventricular focal arrhythmias from intravenous catheter measurements," Expert Systems, vol. 26, no. 1, pp. 70-81, 2009.

[43] T. Kohonen, Self-Organizing Maps, vol. 30 of Springer Series in Information Sciences, Springer, Berlin, Germany, 1995. 
[44] H. Merdun, "Self-organizing map artificial neural network application in multidimensional soil data analysis," Neural Computing and Applications, vol. 20, no. 8, pp. 1295-1303, 2011.

[45] M. Y. Kiang, U. R. Kulkarni, and R. D. St Louis, "Circular/wraparound self-organizing map networks: an empirical study in clustering and classification," Journal of the Operational Research Society, vol. 52, no. 1, pp. 93-101, 2001.

[46] P. C. Dinsmore and J. Cabanis-Brewin, The AMA Handbook of Project Management, Amacom, 2006.

[47] K. Nasrollahzadeh and M. M. Basiri, "Prediction of shear strength of FRP reinforced concrete beams using fuzzy inference system," Expert Systems with Applications, vol. 41, no. 4, part 1, pp. 1006-1020, 2014.

[48] T. Nakashima-Paniagua, J. Doucette, and W. Moussa, "Fabrication process suitability ranking for micro-electro-mechanical systems using a fuzzy inference system," Expert Systems with Applications, vol. 41, no. 9, pp. 4123-4138, 2014.

[49] G. Xiong, D. Shi, and X. Duan, "Enhancing the performance of biogeography-based optimization using polyphyletic migration operator and orthogonal learning," Computers and Operations Research, vol. 41, pp. 125-139, 2014.

[50] D. Simon, "Biogeography-based optimization," IEEE Transactions on Evolutionary Computation, vol. 12, no. 6, pp. 702-713, 2008.

[51] Y.-J. Zheng, H.-F. Ling, H.-H. Shi, H.-S. Chen, and S.-Y. Chen, "Emergency railway wagon scheduling by hybrid biogeography-based optimization," Computers \& Operations Research, vol. 43, pp. 1-8, 2014. 


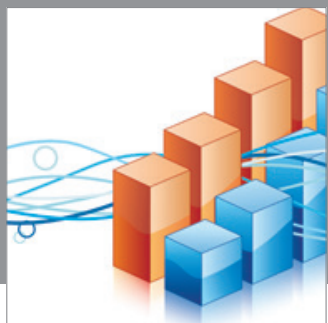

Advances in

Operations Research

mansans

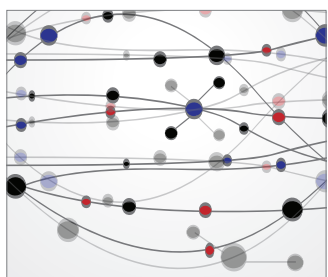

The Scientific World Journal
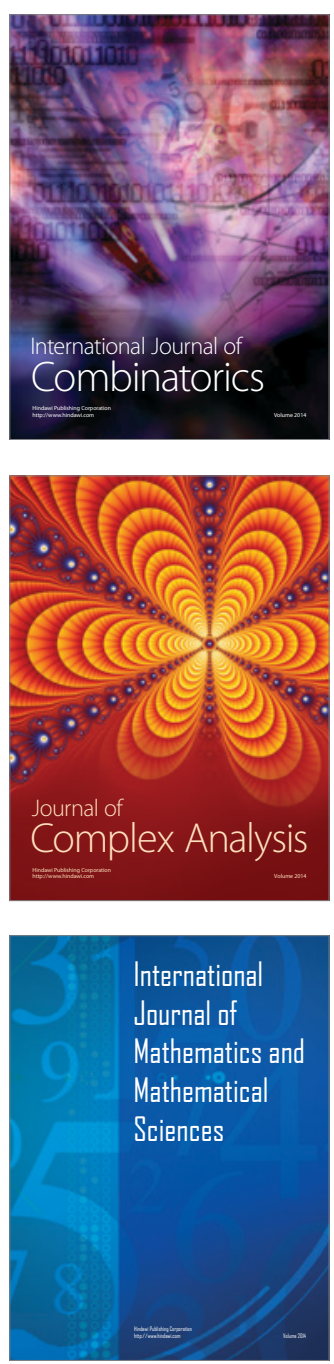
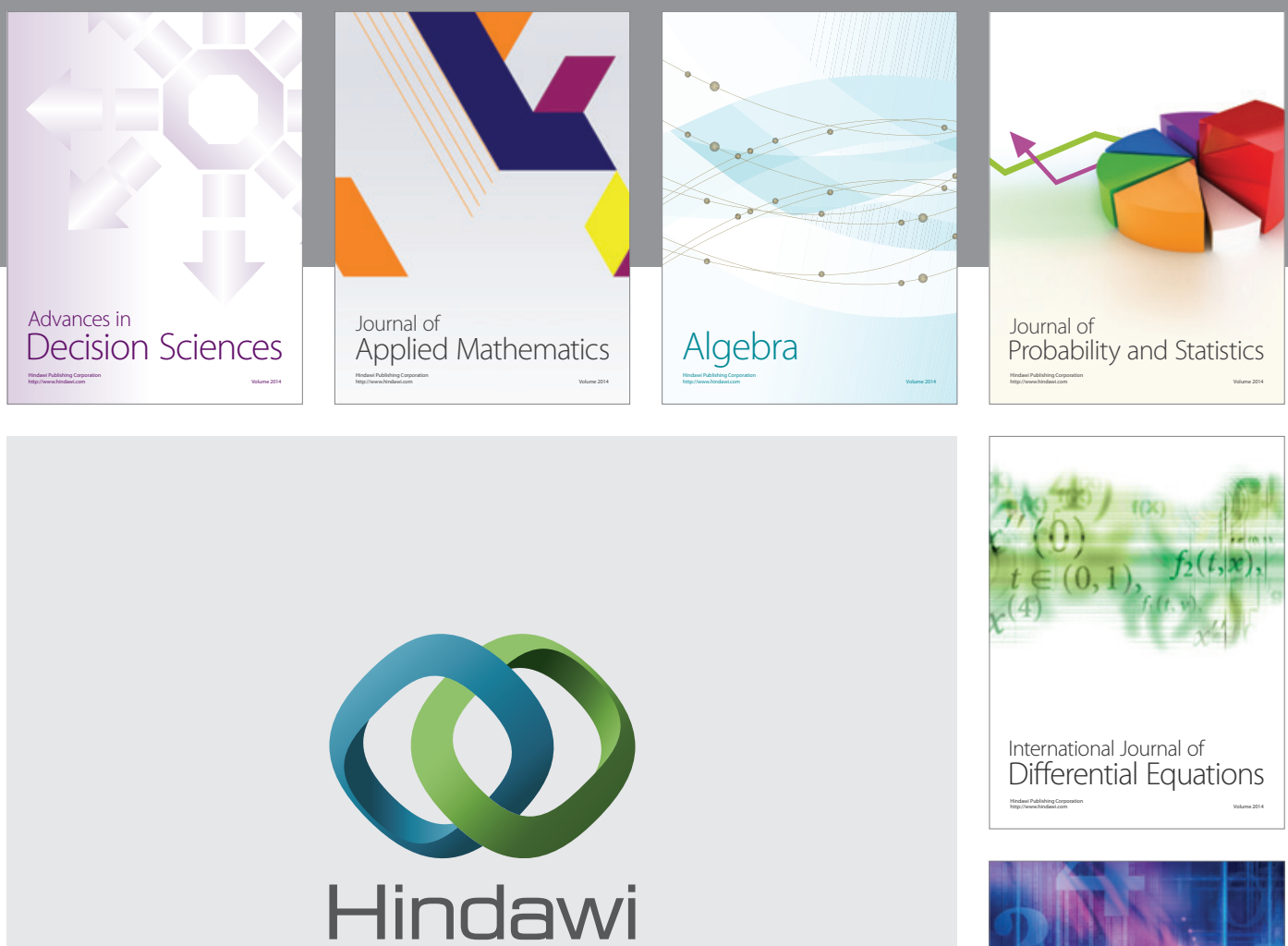

Submit your manuscripts at http://www.hindawi.com
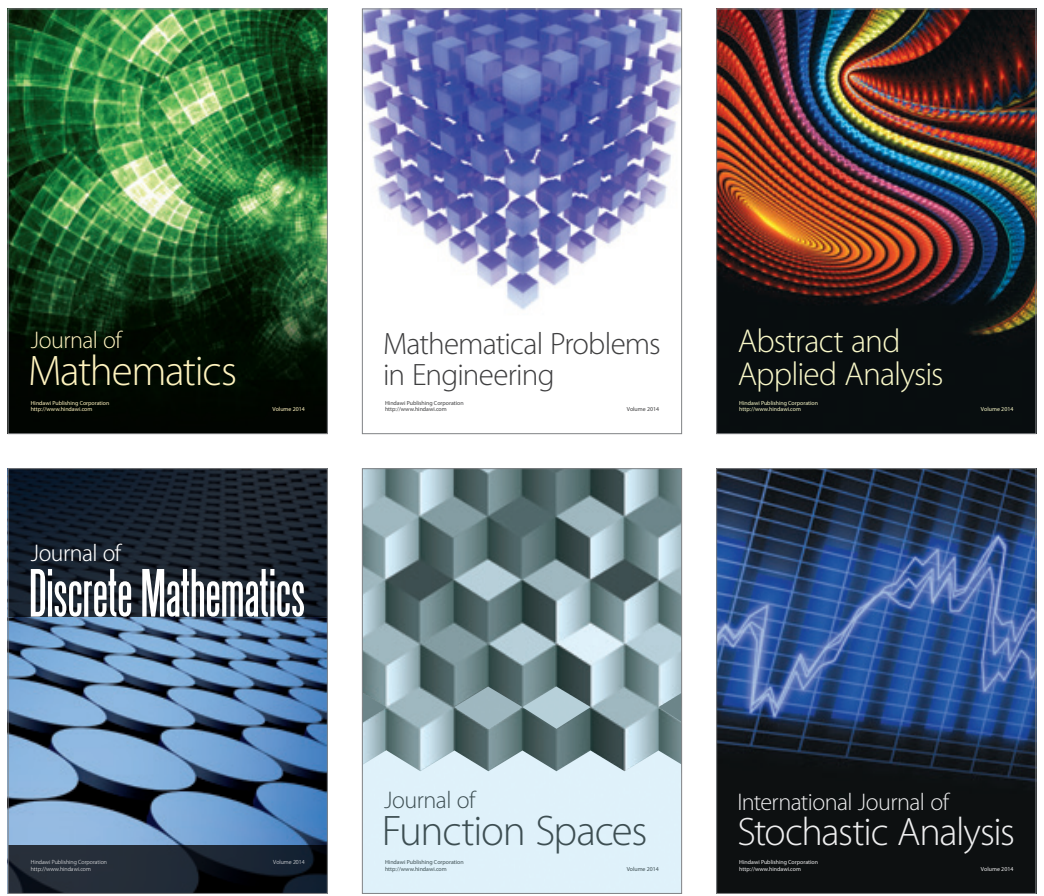

Journal of

Function Spaces

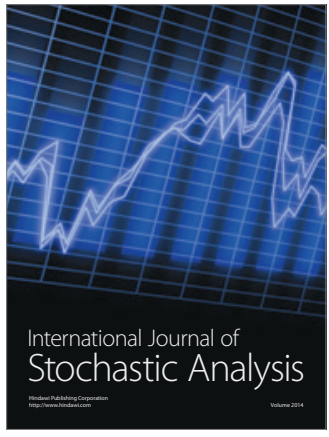

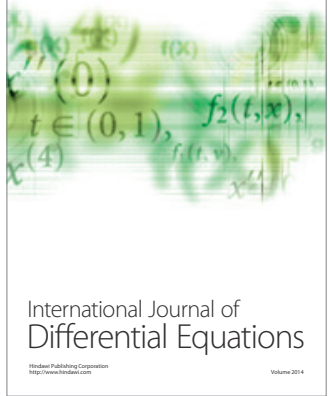
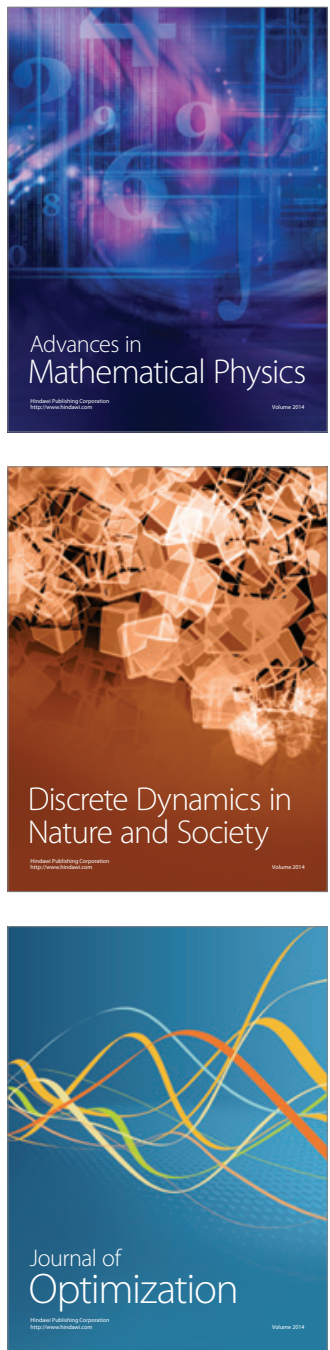